\title{
Intramodal blocking in honeybees
}

\author{
P. A. COUVILLON, LIANNE ARAKAKI, and M. E. BITTERMAN \\ University of Hawaii, Honolulu, Hawaii
}

\begin{abstract}
Foraging honeybees were trained in a concurrent blocking design with a compound stimulus (AX) reinforced and one of its components (A) either reinforced for a blocking group or nonreinforced for a control group. In Experiment 1, a compound of two colors was used; in Experiment 2, a compound of two odors was used; in Experiment 3, a color-position compound, with position defined in terms of proximity to a distinctive visual landmark, was used; and, in Experiment 4, an odor-position compound was used. In each of the first three experiments, the blocking group responded less than did the control group in a subsequent test with $\mathrm{X}$; in the fourth experiment, the two groups did not differ. The results are in accord with expectations based on those of previous experiments with honeybees in which the independence assumption was found to hold for intermodal compounds but not for intramodal compounds.
\end{abstract}

Experiments on the conditioning of color-odor compounds in honeybees (Couvillon \& Bitterman, 1987, 1988, 1989) have given no reason to question the parsimonious independence assumption of traditional continuity theory (Hull, 1929; Spence, 1936) - the assumption that the components of a compound stimulus gain or lose associative strength independently with reinforcement or nonreinforcement of the compound. Perhaps the most compelling indication of independence in the compound conditioning of honeybees has come from recent work by Funayama, Couvillon, and Bitterman (1995), who, in a series of six blocking experiments, failed to find any evidence that the conditioning of either component of a color-odor compound was influenced by the associative strength of the other. In the vertebrate literature, the independence assumption has long been rejected in favor of the assumption that the components of a compound stimulus compete for associative strength (Rescorla \& Wagner, 1972) or for attention (Sutherland \& Mackintosh, 1971), and the color-odor results for honeybees are especially interesting because they are almost alone in pointing to a difference in the learning of honeybees and vertebrates (Bitterman, 1988, 1996). From some experiments with other compounds, however, it appears that the difference may be less profound than the color-odor results suggest.

In the earliest of those experiments, positive intradimensional transfer - a vertebrate phenomenon understandable in terms of competition for attention (Shepp \& Eimas, 1964) - was found for color-position compounds,

This work was supported in part by Grant IBN-9308132 from the National Science Foundation and in part by Research Centers for Minority Institutions Grant RR03061 from the National Institutes of Health. For assistance in data collection, the authors are indebted to Joseph Tolentino, whose participation was made possible by a Research Experience for Undergraduates supplement to the NSF grant, and to Robert Hsiung, a Howard Hughes Medical Institute Undergraduate Research Fellow. Correspondence should be addressed to M. E. Bitterman, Békésy Laboratory of Neurobiology, 1993 East-West Rd., Honolulu, HI 96822 (e-mail: jeffb@pbrc.hawaii.edu). although not for color-odor compounds (Klosterhalfen, Fischer, \& Bitterman, 1978). Foragers trained first to choose between color-position compounds, with one of the dimensions relevant and the other irrelevant, and then in a second such problem with new colors and new positions, performed better in the second problem when the relevant dimension was the same in the two problems than when it was not. After training in a color-odor problem, performance in a second problem with new colors and odors was the same whether or not the relevant dimension was the same in the two problems.

Color-position and color-odor compounds have been found to function differently also in overshadowing experiments. Honeybees trained in a choice problem with color and position confounded fone color always in one position reinforced vs. a second color always in a second position nonreinforced) learned less about the colors and positions than did control animals trained with one of the dimensions relevant and the other irrelevant (Couvillon, Klosterhalfen, \& Bitterman, 1983). The results of a subsequent experiment of the same design with color-odor compounds proved to be perfectly compatible with the independence assumption (Couvillon \& Bitterman, 1989).

How can the different results for color-position and color-odor compounds be understood? With position assumed to be visually determined (Huber, Couvillon, \& Bitterman, 1994) and color-position compounds treated as intramodal compounds, the hypothesis may be considered that independence is less likely in intramodal compounds than in intermodal compounds. Evidence in support of the modality hypothesis is provided by work on learning in honeybees as a function of amount and probability of reward (Couvillon, Mateo, \& Bitterman, 1996). In that work, learning about the color of a target, but not about its odor, was found to be impaired by the presence of a small white dot used to mark the location of reward on the target-overshadowing in dot-color (intramodal) compounds but not in dot-odor (intermodal) compounds. 
Given the persistent failure of Funayama et al. (1995) to demonstrate blocking in color-odor compounds, support for the modality hypothesis may be found also in a demonstration of blocking by Smith and Cobey (1994), who conditioned proboscis extension in harnessed foragers to binary odor mixtures; the conditioning of one component of a mixture was impaired by prior conditioning of the other. Here, however, the argument carries somewhat less conviction, because different methods were used in the two investigations. Smith and Cobey's success, as compared with the failure of Funayama et al., who worked with free-flying foragers, might mean only that proboscis-extension conditioning is a more powerful method. Unfortunately, it is not possible to use that method to look for blocking in color-odor compounds, because proboscis extension in harnessed honeybees is not elicited by visual stimuli. Instead, our strategy in the present experiments was to look for blocking in free-flying foragers trained with intramodal compounds rather than with intermodal compounds.

The potentially powerful "concurrent blocking" design employed in our experiments is one of several that yielded only negative results for color-odor compounds in the series of experiments by Funayama et al. (1995). In symbolic terms, the procedure is for a blocking group to be trained concurrently with $\mathrm{AX}+, \mathrm{A}+$, and $\mathrm{B}-$, and for a control group to be trained with $\mathrm{AX}+, \mathrm{A}-$, and $\mathrm{B}+$, after which both groups have an extinction test with $X$. $B+$ trials for the control group equate the number of reinforcements; $\mathrm{B}-$ trials for the blocking group equate the number of nonreinforcements and experience with the stimuli. The prediction of Rescorla-Wagner theory is that $\mathrm{A}+$ trials for the blocking group will serve to reduce the associative strength acquired by $\mathrm{X}$ on $\mathrm{AX}+$ trials, and $A-$ trials for the control group will serve to increase the associative strength acquired by $\mathrm{X}$ on $\mathrm{AX}+$ trials, with the result that, in the test with $X$, the blocking group will respond less than the control group. The prediction of attention theory is that attention to $\mathrm{X}$ will be weakened by $A+$ trials and enhanced by $A$ - trials, again with the result that the blocking group will respond less to $X$ in the test. It may be well to emphasize that this is not a "pure" blocking design, because a difference between the groups might be due, as both the Rescorla-Wagner and attentional theories suggest, to some combination of impairment in the blocking group and enhancement in the control group, but there does not seem to be a blocking design with a theoretically neutral control procedure that is entirely satisfactory. What is critical for our purposes, in any case, is that a difference between the groups in response to $\mathrm{X}$ would contradict the independence assumption, which gives no reason to expect one.

\section{EXPERIMENT 1}

Our purpose in Experiment 1 was to look for evidence of blocking in a compound of two colors. In the previous work by Funayama et al. (1995) that provided the model,
$\mathrm{AX}$ was a color-odor compound ( $\mathrm{A}$ a color and $\mathrm{X}$ an odor, or the opposite). In this experiment, both $A$ and $X$ were colors.

\section{Method}

Subjects. The subjects were 32 foraging honeybees, all experimentally naive, from our own hives situated near the laboratory. They were assigned at random to blocking and control groups of 16 subjects each.

Procedure. The subjects of this experiment and all following experiments were trained individually, each in a single session lasting several hours. In the pretraining, a forager was selected at random from a group of foragers at a feeding station providing $10 \%-12 \%$ sucrose solution, picked up in a match box, carried to a laboratory window, and set down at a large drop of $50 \%$ sucrose solution on a pretraining target placed on the deep sill of the window. The subject was marked with a spot of colored lacquer as it fed to repletion, after which it left for the hive. Typically, the subject would return to the laboratory after a few minutes, continuing to fly back and forth between the hive and the window as long as sucrose was available there. If the marked subject did not return after its first placement, it was taken again from the feeding station (where it usually could be found) to the pretraining target and permitted again to feed to repletion. More than two placements were rarely required. The pretraining ended with the subject's first return to the window of its own accord.

The targets were plastic petri dishes, $5.5 \mathrm{~cm}$ in diameter, with gray covers. To some covers, two strips of colored plastic (blue and yellow) were affixed at the locations diagrammed in Figure 1; to other covers, only a single colored strip (blue or yellow) was affixed at one or the other of the two locations. Blue was always to the left for half the subjects in each group and always to the right for the rest. Each target used on any visit was drawn at random from a large set of identical targets, to which it was returned after washing at the end of the visit; the purpose of the procedure (used routinely in this laboratory) is to randomize irrelevant stimuli.

In pretraining and on 8 of 16 training visits, there was a single target labeled with both colors (AX). The target was centered on the window sill, and centered on the cover of the target was a $100-\mu 1$ drop of 50\% sucrose solution, from which there was feeding to repletion. On the remaining 8 visits, intermixed with the first 8 in balanced quasi-random order, the subjects were trained to discriminate between a plain gray target $(B)$ and a target labeled with only one of the colors (A), blue for half the subjects in each group and yellow for the rest. For the subjects of the blocking group, the gray tar-

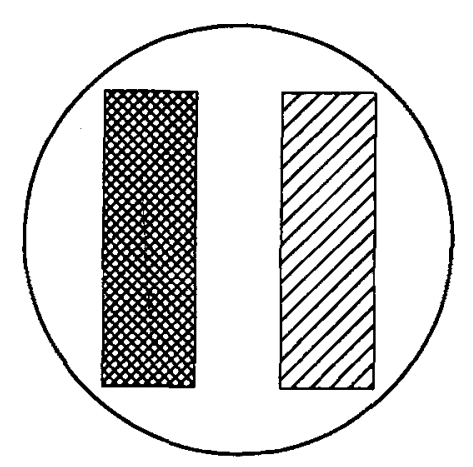

Figure 1. Diagram of the targets (covered plastic petri dishes, $5.5 \mathrm{~cm}$ in diameter, their covers gray in color) used in Experiment 1. On some covers, there were two strips of colored plastic (blue and yellow) at the locations shown; on other covers, only a single strip was affixed at one of the locations, or none. 


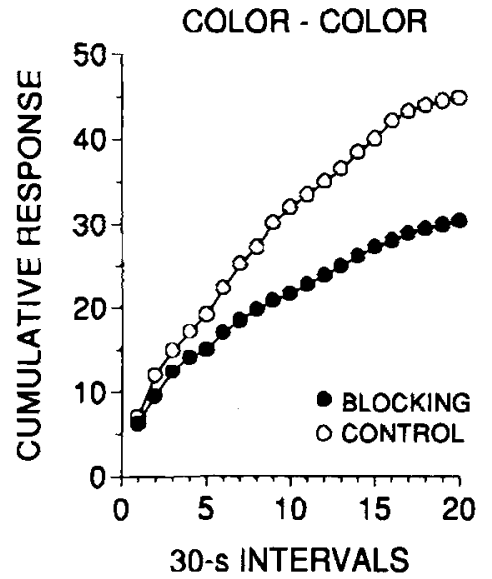

Figure 2. Test performance of the blocking and control groups in Experiment 1 plotted in terms of the mean cumulative number of responses in successive 30 -sec intervals.

get was negative and A was positive; the opposite was true for the subjects of the control group. As in the previous color-odor work, a powerful discrimination procedure developed in earlier work with honeybees (Couvillon \& Bitterman, 1980) was used to ensure substantial experience with the negative alternative. Each of the 8 discrimination-training visits began with presentation of the negative target containing a drop of water (unacceptable to the animal and distinguishable from the sucrose solution only by taste); then, after a set delay (1 min on the first visit, $1.5 \mathrm{~min}$ on the second, and $2 \mathrm{~min}$ on the rest), the negative target was replaced by the positive target containing a drop of $50 \%$ sucrose solution, from which there was feeding to repletion. On the visit following the last training visit, there was an extinction test with a target that was labeled with the color $(X)$ not used in the discriminative training. The target contained a drop of water, and all contacts with the target in a 10-min period were recorded. The familiar behavior of a forager in such a test is to land on the target, taste the water, fly up from the target, land again, fly up again with or without tasting the water, and so forth, the interval between successive contacts with the target increasing progressively.

\section{Results}

In Figure 2, the performance of the blocking and control groups in the extinction test with $\mathrm{X}$ is plotted (as is conventional in our experiments) in terms of the mean cumulative number of responses in successive $30-\mathrm{sec}$ intervals. The curves show less responding in the blocking group than in the control group. An analysis of variance (ANOVA) based on the uncumulated data yielded a significant group effect $[F(1,30)=6.72, p<.05]$ and a significant change in responding over 2.5 -min blocks $[F(3,90)=50.21, p<.01]$, without a significant group $X$ block interaction $[F(3,90)=1.62, p>.05]$. The results contradict the independence assumption.

\section{EXPERIMENT 2}

In Experiment 2, we looked again for evidence of intramodal blocking in free-flying honeybees, but with a compound of two odors rather than of two colors. Actually, there were two such experiments. One of them (Ex- periment 2A) was with geraniol and hexanol, odors used by Smith and Cobey (1994) to demonstrate blocking in proboscis-extension conditioning. The second experiment (Experiment 2B) was with geraniol and peppermint, the two odors we have used extensively both in proboscis-extension conditioning (e.g., Hoban, Couvillon, \& Bitterman, 1996) and in prior work with free-flying honeybees.

\section{Method}

Subjects. The subjects were 48 foraging honeybees, all experimentally naive, from our own hives situated near the laboratory. They were assigned at random to four groups of 12 subjects each: a blocking group and a control group for Experiment $2 \mathrm{~A}$, and a blocking group and a control group for Experiment 2B.

Procedure. The targets were plastic petri dishes, $5.5 \mathrm{~cm}$ in diameter, with gray covers. Drilled in the cover of each dish, $6 \mathrm{~mm}$ from its outer circumference, was a circle of eight equally spaced holes, $5 \mathrm{~mm}$ in diameter. The dishes contained pieces of cotton batting that could be impregnated as required with the odors of geraniol, hexanol, or both in Experiment $2 \mathrm{~A}$, and with geraniol, peppermint, or both in Experiment $2 \mathrm{~B}$. When two odors were used, the batting on one side of the target was impregnated with one of the odors and the batting on the other side with the other odor. Odors were balanced in the design, which was exactly the same as that of Experiment 1 except that $A$ and $X$ were odors instead of colors, one of the two odors serving as $A$ for half the animals in each group and as $\mathrm{X}$ for the rest. $\mathrm{B}$ was again a plain gray, unscented target.

\section{Results}

In Figures 3 and 4 , the performance of the blocking and control groups in the extinction test with $X$ is plotted in terms of the mean cumulative number of responses in successive $30-\mathrm{sec}$ intervals. Figure 3 shows that there was less responding in the blocking group than in the control group of Experiment $2 \mathrm{~A}$ (training with geraniol and hexanol). An ANOVA yielded a significant group effect $[F(1,22)=5.07, p<.05]$, a significant change in responding over 2.5 -min blocks $[F(3,66)=37.52$, $p<.01]$, and a significant group $\times$ block interaction $[F(3,66)=3.16, p<.05]$. Figure 4 shows that there was also less responding in the blocking group than in the control group of Experiment $2 \mathrm{~B}$ (training with geraniol

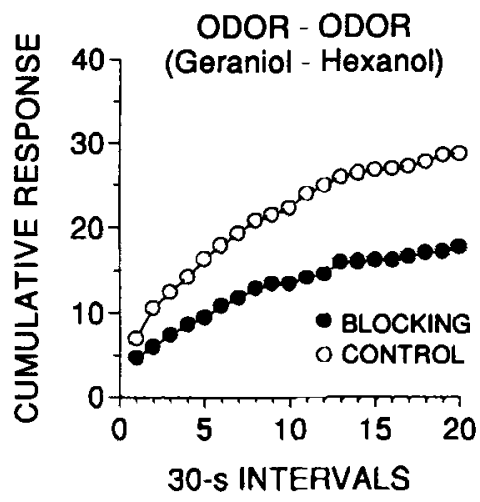

Figure 3. Test performance of the blocking and control groups in Experiment $2 \mathrm{~A}$ plotted in terms of the mean cumulative number of responses in successive 30 -sec intervals. 


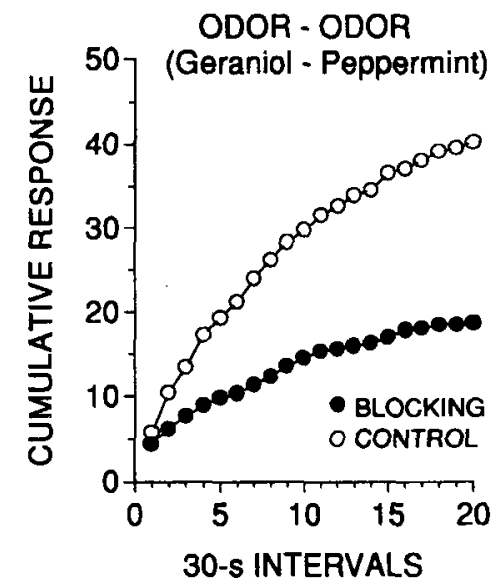

Figure 4. Test performance of the blocking and control groups in Experiment 2B plotted in terms of the mean cumulative number of responses in successive 30 -sec intervals.

and peppermint). An ANOVA here, too, yielded a significant group effect $[F(1,22)=10.29, p<.01]$, a significant change in responding over 2.5 -min blocks $[F(3,66)=$ $38.74, p<.01]$, and a significant group $\times$ block interaction $[F(3,66)=3.52, p<.05]$. These blocking results for two-odor compounds, like those of Experiment 1 for twocolor compounds, contradict the independence assumption.

\section{EXPERIMENT 3}

The first honeybee data to challenge the independence assumption came from experiments that showed positive intradimensional transfer in training with color-position compounds but not with color-odor compounds (Klosterhalfen et al., 1978). In Experiment 3, we looked for colorposition blocking, which would be expected on the modality hypothesis if, in fact, it is correct to treat color-position compounds as intramodal. It is not clear whether, in the earlier work, position was given in relation to the visual surround (place learning) or (literally) to position in the animal's visual field as determined by the animal's orientation (Huber et al., 1994; Wehner, 1981). Here, we chose to work with proximity to a distinctive visual landmark, which, on the basis of previous work, we were confident would have considerable salience for the animals (Couvillon, Leiato, \& Bitterman, 1991). That the landmark we used did, in fact, acquire control of behavior is evidenced by the outcome of the experiment.

\section{Method}

Subjects. The subjects were 32 foraging honeybees, all experimentally naive, from our own hives situated near the laboratory. They were assigned at random to blocking and control groups of 16 subjects each.

Procedure. The targets were drawn from two sets of petri dishes, $5.5 \mathrm{~cm}$ in diameter, with colored covers, those of one set blue and those of the other yellow. For half the animals in each group, yellow was $\mathrm{X}$ and blue was $\mathrm{B}$; the opposite was true for the remaining animals. The landmark (A) was a white wooden block, $11 \mathrm{~cm}$ long and $4 \mathrm{~cm}$ in each of its other dimensions. The procedure was much the same as in Experiments 1 and 2, but some change was necessary to accommodate the fact that the landmark was not an integral target property; its associative strength, therefore, could be modulated only by reward or nonreward on an adjacent target. In the pretraining and on 8 of the subsequent 16 training trials, AX was rewardeda target of one of the colors $(X)$ at the center of the sill with the landmark (A) lying on its long side parallel to the outer edge of the sill at a distance of $1 \mathrm{~cm}$ directly behind the target. On the 8 trials of discriminative training, targets of the alternative color $(B)$ were presented both with the landmark $(\mathrm{AB})$ and without it (B alone). For the blocking animals, $\mathrm{AB}$ was positive and $\mathrm{B}$ negative; the opposite was true for the control animals. The extinction test was with $\mathrm{X}$ alone. The predictions from the Rescorla-Wagner and attention theories are the same as before: The discriminative training should tend to reduce response to $\mathrm{X}$ in the blocking group and to enhance it in the control group. The independence assumption provides no basis for expecting that there will be a difference in the test performance of the two groups.

\section{Results}

In Figure 5, the performance of the blocking and control groups in the extinction test with $\mathrm{X}$ is plotted in terms of the mean cumulative number of responses in successive $30-\mathrm{sec}$ intervals. Again, the curves show less responding in the blocking group than in the control group. An ANOVA yielded a significant group effect $[F(1,30)=4.52, p<.05]$ and a significant change in responding over 2.5 -min blocks $[F(3,90=37.85$, $p<.01]$, without a significant group $\times$ block interaction $[F(3,90)=1.33, p<.05]$. These blocking results, like those of Experiments 1 and 2 for two-color and two-odor compounds, fail to meet expectations based on the independence assumption.

\section{EXPERIMENT 4}

In Experiment 4, we looked for odor-position blocking rather than color-position blocking, with position defined again by proximity to a distinctive visual landmark. If the absence of blocking in previous experiments with color-odor compounds by Funayama et al. (1995)

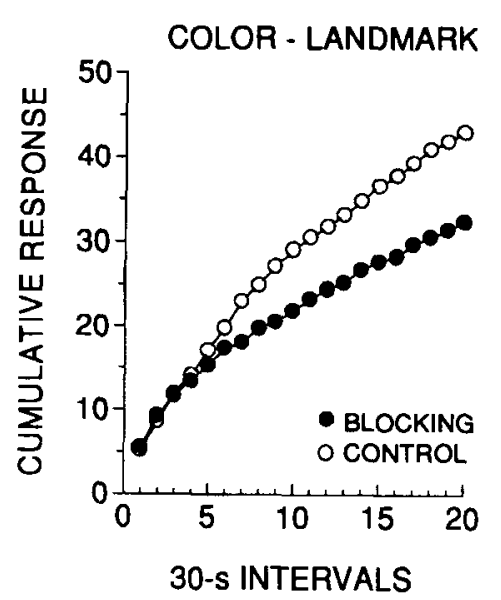

Figure 5. Test performance of the blocking and control groups in Experiment 3 plotted in terms of the mean cumulative number of responses in successive 30-sec intervals. 


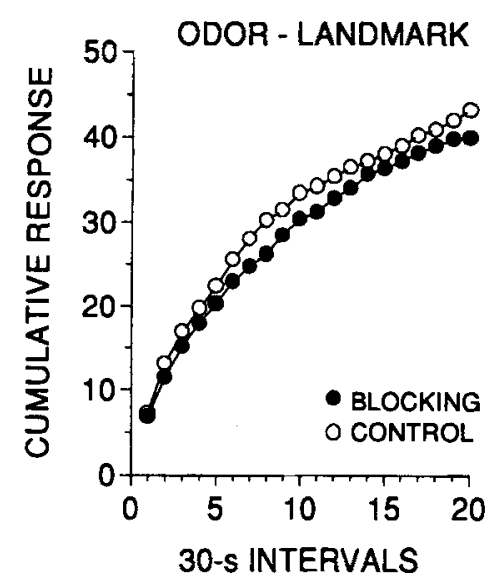

Figure 6. Test performance of the blocking and control groups in Experiment 4 plotted in terms of the mean cumulative number of responses in successive 30-sec intervals.

were due to their intermodality, negative results should be obtained here as well.

\section{Method}

Subjects. The subjects were 32 foraging honeybees, all experimentally naive, from our own hives situated near the laboratory. They were assigned at random to blocking and control groups of 16 subjects each.

Procedure. The design of this experiment was the same as that of Experiment 3, but now the two sets of targets, $\mathrm{X}$ and $\mathrm{B}$, had gray covers like those used in Experiment 2. The targets of one set were scented with geraniol and those of the second set with peppermint. For half the animals in each group, $X$ was geraniol and $B$ was peppermint; the opposite was true for the remaining animals. The white wooden block was again the landmark.

\section{Results}

In Figure 6, the performance of the blocking and control groups in the extinction test with $X$ is plotted in terms of the mean cumulative number of responses in successive 30-sec intervals. Here, the curves are very much the same. An ANOVA yielded a significant change in responding over 2.5 -min blocks $[F(3,90)=65.86, p<$ $.01]$, but neither a significant group effect $(F<1)$ nor a significant group $\times$ block interaction $(F<1)$. These results-like those of Funayama et al. (1995) with colorodor compounds, which also failed to show blockingare in accord with the independence assumption.

\section{DISCUSSION}

The results of these experiments fit nicely into the pattern established in previous work on compound conditioning in honeybees. In the experiments with two-color, two-odor, and color-position compounds, the blocking groups responded less on test trials with the critical components than did the control groups (outcomes that contradict the independence assumption), although the performance of the blocking and control groups did not differ in the odor-position experiment. The pattern in the results for honeybees-if position is given visually - is that the independence assumption holds for intermodal compounds but not for intramodal compounds.

How is the pattern to be explained? Could the learning process be different for intramodal compounds than for intermodal compounds? A more reasonable interpretation seems to be that independence is promoted, not by heteromodality per se, but by the distinguishability of components, which is more likely to be impaired by afferent interaction in intramodal compounds than in intermodal compounds. In the vertebrate literature, of course, reports of intermodal blocking are common, but much the same explanation (greater afferent interaction in intramodal compounds) has been considered for the fact that summation of associative strengths has been more difficult to demonstrate in intramodal compounds than in intermodal compounds (Kehoe, Horne, Horne, \& Macrae, 1994; Rescorla \& Coldwell, 1995). Rescorla and Coldwell found no evidence of summation in a simultaneous compound of two visual components, but they did find summation when, in an effort to reduce afferent interaction, the components were presented successively rather than simultaneously, and a parallel effort to modulate intramodal blocking in honeybees might be instructive. On the assumption that spatial disaggregation functions like temporal disaggregation, a useful strategy in the work with honeybees might be, for example, to repeat Experiment 3 with increasing distance between target and landmark. Curtailment of blocking under those circumstances would strengthen the impression that distinguishability of components rather than heteromodality per se is critical for independence, and that we are not dealing here with a difference in the learning of honeybees and vertebrates, but only in their perception.

The two principal alternatives to the independence assumption that have been offered in the vertebrate literature are the assumptions of competition for associative strength (Rescorla \& Wagner, 1972) and competition for attention (Sutherland \& Mackintosh, 1971). The RescorlaWagner theory is simpler than attention theory (which provides for learned changes in attention as well as in associative strength), and its implications are clearer; however, it does not now seem to be very useful, at least for honeybees. It predicts a pattern of results quite different from that obtained in the dot-overshadowing experiments (Couvillon et al., 1996), and it provides no basis for understanding the lack of blocking either in the six colorodor experiments by Funayama et al. (1995) or in the fourth (odor-position) experiment of the present series. The salience of compound-unique components generated by afferent interaction might be much less in the intermodal case than in the intramodal case, but intermodal blocking still would be expected. Simulations with a compound- unique component (Q)-AXQ+/ $\mathrm{A}+/ \mathrm{B}-$ versus $\mathrm{AXQ}+/ \mathrm{A}-/ \mathrm{B}+$ training - actually show that, as the salience of $Q$ increases, the asymptotic associative strength of $X$ declines in the control group and remains essentially at zero in the blocking group; that is, the predicted difference between the two groups, which 
is the measure of blocking, declines (although some difference remains).

Rescorla-Wagner theory is silent on the facts of intradimensional transfer in honeybees (Klosterhalfen et al., 1978), which can be dealt with in attentional terms on the assumption that competition for attention is less in color-odor compounds than in color-position compounds, giving the impression of independence in the intermodal case. That color-odor compounds are far from immune to afferent interaction is suggested, however, by work on conditional discrimination (Couvillon \& Bitterman, 1988): How otherwise, except in the vaguest conceptual terms, are we to explain, for example, that honeybees can be trained - although with a great deal of difficulty - to choose green-peppermint and blue-geraniol targets (reinforced) rather than green-geraniol and blue-peppermint targets (nonreinforced)? It should be noted, too, that the blocking found with compounds of two colors in Experiment 1 and with two odors in Experiment 2 would have to be attributed to competition for attention, not $b e$ tween dimensions--Sutherland and Mackintosh's (1971) "analyzers"- -but within dimensions. When this evidence of competition within dimensions (intradimensional competition) is added to the evidence of competition between dimensions (interdimensional competition) provided by the work on dimensional transfer in the discrimination of color-position compounds, the problem of interpretation is complicated considerably. A formal attention theory that will permit rigorous derivation of all the available honeybee data remains to be invented.

\section{REFERENCES}

BitTerman, M. E. (1988). Vertebrate-invertebrate comparisons. In H. J. Jerison \& I. Jerison (Eds.), Intelligence and evolutionary biology (pp. 251-276). Berlin: Springer-Verlag.

BitTERman, M. E. (1996). Comparative analysis of learning in honeybees. Animal Learning \& Behavior, 24, 123-141.

Couvillon, P. A., \& Bitterman, M. E. (1980). Some phenomena of associative learning in honeybees. Journal of Comparative \& Physiological Psychology, 94, 878-885.

Couvillon, P. A., \& Bitterman, M. E. (1987). Discrimination of color-odor compounds by honeybees: Tests of a continuity model. Animal Learning \& Behavior, 15, 218-227.

Couvillon, P. A., \& Bitterman, M. E. (1988). Compound-component and conditional discrimination of colors and odors by honeybees: Further tests of a continuity model. Animal Learning \& Behavior, 16 , 67-74.
Couvillon, P. A., \& Bitterman, M. E. (1989). Reciprocal overshadowing in the discrimination of color-odor compounds by honeybees: Further tests of a continuity model. Animal Learning \& Behavior, 17, 213-222.

Couvillon, P. A., Klosterhalfen, S., \& Bitterman, M. E. (1983). Analysis of overshadowing in honeybees. Journal of Comparative Psychology, 97, 154-166.

Couvillon, P. A., Leiato, T. G., \& Bitterman, M. E. (1991). Learning by honeybees (Apis mellifera) on arrival and departure from a feeding place. Journal of Comparative Psychology, 105, 177-184.

Couvillon, P. A., Mateo, E. T., \& Bitterman, M. E. (1996). Reward and learning in honeybees: Analysis of an overshadowing effect. Animal Learning \& Behavior, 24, 19-27.

Funayama, E. S., Couvillon, P. A., \& Bitterman, M. E. (1995). Compound conditioning in honeybees: Blocking tests of the independence assumption. Animal Learning \& Behavior, 23, 429-437.

Hoban, J. S., Couvillon, P. A., \& Bitterman, M. E. (1996). Odorpreference in honeybees as a function of amount of reward: Tests of two hypotheses. Journal of Insect Behavior, 9, 121-132.

Huber, B., Couvillon, P. A., \& Bitterman, M. E. (1994). Place and position learning in honeybees (Apis mellifera). Journal of Comparative Psychology, 108, 213-219.

HulL, C. L. (1929). A functional interpretation of the conditioned reflex. Psychological Review, 36, 498-511.

Kehoe, E. J., Horne, A. J., Horne, P. S., \& Macrae, M. (1994). Summation and configuration between and within sensory modalities in classical conditioning of the rabbit. Animal Learning \& Behavior, 22, 19-26.

Klosterhalfen, S., Fischer, W., \& Bitterman, M. E. (1978). Modification of attention in honeybees. Science, 210, 1241-1243.

ResCorla, R. A., \& ColDWELl, S. E. (1995). Summation in autoshaping. Animal Learning \& Behavior, 23, 314-326.

RESCORLA, R. A., \& WAGNER, A. R. (1972). A theory of Pavlovian conditioning: Variations in the effectiveness of reinforcement and nonreinforcement. In A. H. Black \& W. F. Prokasy (Eds.), Classical conditioning II: Current research and theory (pp. 64-99). New York: Appleton-Century-Crofts.

SHEPP, B. E., \& EIMAS, P. D. (1964). Intradimensional and extradimensional shifts in the rat. Journal of Comparative \& Physiological Psychology, 57, 357-361.

SMith, B. H., \& COBEY, S. (1994). The olfactory memory of the honeybee Apis mellifera. Journal of Experimental Biology, 195, 91-108.

SPENCE, K. W. (1936). The nature of discrimination learning in animals. Psychological Review, 43, 427-449.

Sutherland, N. S., \& MaCKintosh, N. J. (1971). Mechanisms of animal discrimination learning. New York: Academic Press.

WEHNER, R. (1981). Spatial vision in arthropods. In H. Autrum (Ed.), Comparative physiology and evolution of vision in invertebrates: C. Invertebrate visual centers and behavior II (pp. 287-616). Berlin: Springer-Verlag.

(Manuscript received July l, 1996; revision accepted for publication November 5,1996 .) 